\title{
Particle acceleration and jet dynamics in Centaurus A
}

\author{
Martin J. Hardcastle ${ }^{\mathrm{a}}$, Diana M. Worrall ${ }^{\mathrm{a}}$, Ralph P. Kraft ${ }^{\mathrm{b}}$, W.R. Forman ${ }^{\mathrm{b}}$, C. Jones ${ }^{\mathrm{b}}$ and S.S. Murray $^{\mathrm{b}}$ \\ ${ }^{a}$ Department of Physics, University of Bristol, Tyndall Avenue, Bristol BS8 1TL, UK

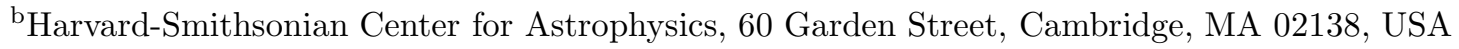

New multi-epoch Chandra observations, combined with multi-epoch, high-dynamic range VLA data, have allowed us to relate the radio and X-ray structure of the jet in Cen A to the jet dynamics. We present evidence that a significant fraction of the structure in the Cen A X-ray jet is due to particle acceleration at the interaction between the jet flow and internal galactic material.

\section{Low-luminosity jets}

The general properties of jets in FRI radio galaxies are now comparatively well understood. On parsec scales, jets in FRIs are similar to those in the more powerful FRIIs; they are well collimated and relativistic. On scales of a few kpc, however, the FRI jets disrupt as they come into pressure balance with the external medium, becoming much less well collimated and less efficient as energy transport mechanisms, and producing the characteristic bright, broad jet emission seen on arcsecond scales with instruments such as the VLA. The jets decelerate from relativistic to subrelativistic speeds on scales of a few kpc, showing significant transverse velocity structure [1]. The deceleration is likely to be a result of entrainment of external material, and the jet is stabilized by the strong pressure gradient in the centre of the host galaxy [1-3]. It is now possible to make detailed quantitative models of these jets, which agree well with observation (e.g. [3]).

\section{Beyond the standard picture}

These jet models do not tell us why the jets are observed: how do the jet dynamics give rise to the population of high-energy particles responsible for the observed synchrotron emission? The energy required for particle acceleration must certainly come from the bulk kinetic energy of the jet, but the mechanism the jets use to tap this energy is not clear.

In recent years X-ray observations of jets have
Table 1

FRI radio galaxies with known X-ray jets

\begin{tabular}{llr}
\hline Name & $z$ (distance) & Reference \\
\hline Cen A & $(3.4 \mathrm{Mpc})$ & {$[6]$} \\
M87 & $(20 \mathrm{Mpc})$ & {$[7]$} \\
B2 0206+35 & 0.0375 & {$[4]$} \\
B2 0755+37 & 0.0413 & {$[4]$} \\
3C 66B & 0.0215 & {$[5]$} \\
3C 31 & 0.0169 & {$[8]$} \\
3C 129 & 0.0208 & {$[9]$} \\
M84 & $(20 \mathrm{Mpc})$ & {$[10]$} \\
3C 270 & $(40 \mathrm{Mpc})$ & {$[11]$} \\
NGC 315 & 0.0167 & {$[12]$} \\
3C 15 & 0.0730 & {$[13]$} \\
\hline
\end{tabular}

shown that X-ray emission from the inner jets of FRIs is common, and perhaps even universal [4]. Table 1 lists the FRI X-ray jets known at the time of writing. In every well-studied case, the X-ray spectrum, combined with the available data at lower frequencies, is consistent with a synchrotron origin for the X-rays [5]. In some senses this is disappointing, since inverse-Compton emission would have given us direct information about physical conditions and/or bulk speeds in the jets. However, synchrotron X-rays do give us important information about the locations of particle acceleration in the jets. In magnetic fields close to the standard equipartition values, $\mathrm{X}$-ray-emitting electrons have energies of up to tens of $\mathrm{TeV}$, and synchrotron loss timescales $(E / \dot{E})$ of the order 
of tens of years. Even at the mildly relativistic speeds inferred from jet observations, the electrons can travel only a few parsecs from their point of origin before their characteristic emission frequency falls below the X-ray band. Observations of synchrotron X-rays therefore tell us where particle acceleration is happening now, and there is a real possibility of observing variability in X-ray structures on timescales of years as a result of synchrotron losses. By contrast, in the radio waveband, the synchrotron loss timescale is $10^{4}-10^{5}$ years, and so downstream advection, as well as particle acceleration, shapes the observed structure of the jet, while variability observed in the radio must be a result of other processes.

The observation that X-ray synchrotron emission is associated with the inner few kpc of many FRI jets therefore implies that particle acceleration is taking place in these regions, and - purely on the basis of the spatial coincidence - it can be suggested that the particle acceleration is associated with the strong jet deceleration that FRI jet models imply in these regions [8]. However, the spatial resolution even of Chandra $\left(\sim 0.5^{\prime \prime}\right)$ at the distances of most FRI sources with jet detections is of the order of hundreds of pc (Table 1). It is therefore impossible to say whether the particle acceleration takes place in discrete regions, for example via the standard first-order Fermi process at shocks, or is diffused throughout the jet (mechanisms for this include second-order Fermi acceleration at turbulence, Alfvénic turbulence, or magnetic field line reconnection).

For these reasons, it is particularly interesting to study Centaurus A, the nearest radio galaxy to us. Its X-ray jet was the first to be discovered [6] and, at Cen A's distance of 3.4 Mpc [14] Chan$d r a$ 's resolution corresponds to about $10 \mathrm{pc}$. As the next nearest radio galaxies are in the Virgo cluster, 7 times further away, Cen A is the only object in which the available spatial resolution is comparable to the X-ray synchrotron loss distance.

\section{Centaurus A}

As Cen A is the closest active galaxy and the closest AGN, and its host NGC 5128 is the clos- est giant elliptical to us, it has been extensively studied with X-ray telescopes, including recent observations with Beppo-SAX [15], Chandra and $X M M-N e w t o n$ [16-19]. Here we shall concentrate on Chandra observations taken in 2002 [19], using the ACIS-S array with the jet near the aim point.

Perhaps surprisingly, detailed, high-dynamicrange radio images of the Cen $\mathrm{A}$ jet were not available in the literature, because the source is only just accessible to the VLA $\left(\delta=-43^{\circ}\right)$. We observed Cen $\mathrm{A}$ with the VLA at $8 \mathrm{GHz}$ in 2002, and, combining these observations with data taken in 1991 and using a technique of baseline-based self-calibration, were able to obtain images with a dynamic range that was an order of magnitude better than had been obtained previously. This had two important results. Firstly, we were able to detect proper motions in some, but not all, of the knots in the jet (Fig. 1), with apparent speeds on the sky corresponding to around $0.5 c$. Secondly, we were able to show that some previously detected faint X-ray knots in the jet (knots BX2, AX6, AX5, AX3, AX2, SX2A, SX2B in Fig. 2) were associated with weak, previously unknown radio knots in our new radio images. Most interesting of all, these weak radio knots with bright $\mathrm{X}$-ray counterparts were clearly stationary or slow-moving compared with the radio features that exhibited significant proper motion. The knots that did exhibit proper motion had radio-to-X-ray flux ratios comparable to that of the jet as a whole; it is important to note that a significant fraction of the $\mathrm{X}$-ray flux from the jet appears to be diffuse (Fig. 3 ) and is not associated with any compact radio features.

\section{Particle acceleration}

The radio-to-X-ray spectra of the radio-faint, $\mathrm{X}$-ray-bright, stationary or slow-moving knots, compared with the spectrum of the extended emission, rule out the possibility that they are simply compressions in the fluid flow, and we can also reject models in which they are related to jet-triggered supernovae. Instead, they must be privileged sites for particle acceleration in the jet. It is therefore very tempting to relate their parti- 


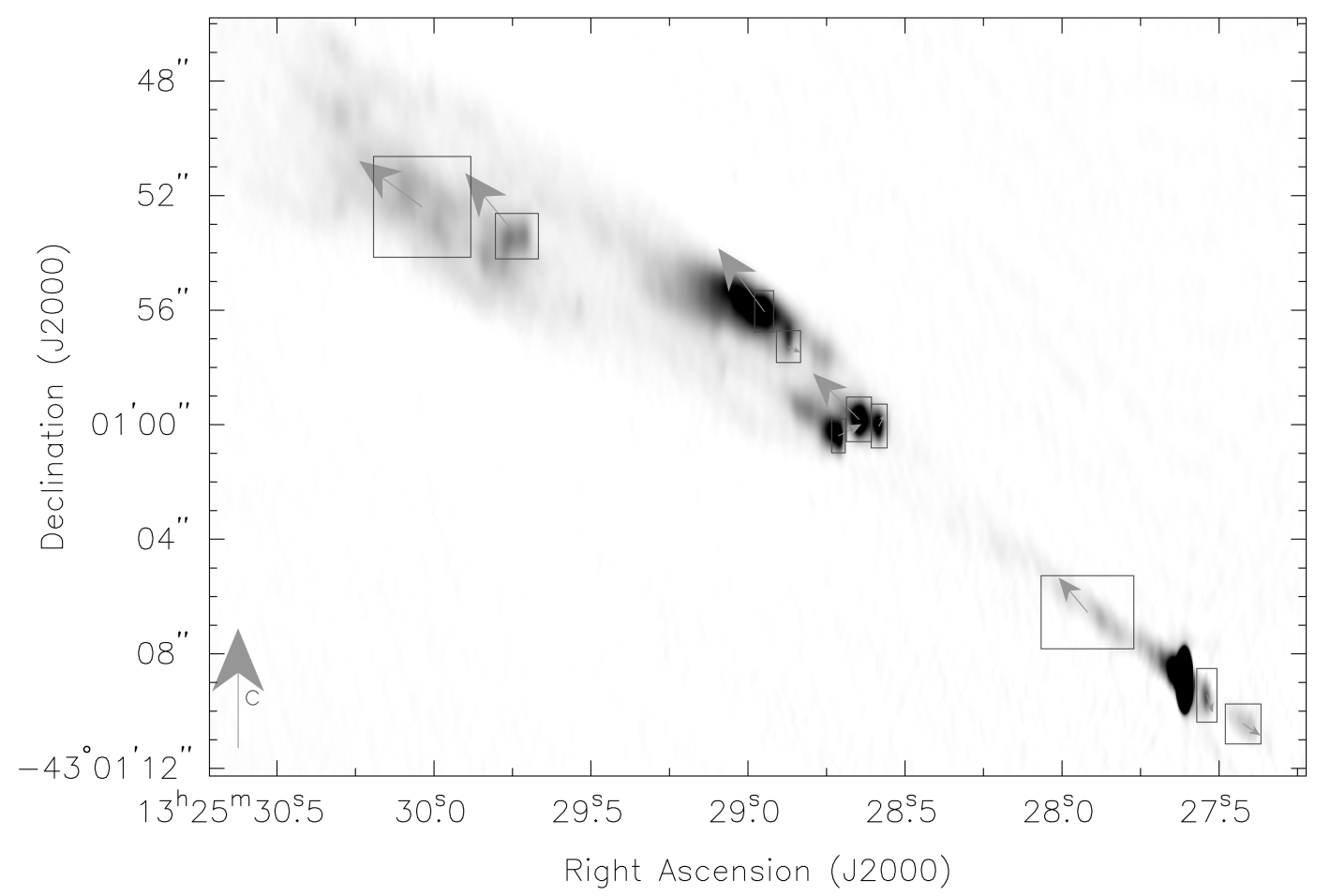

Figure 1. Proper motions in the jet of Centaurus A. Vectors show the directions of the observed motions. Note that some knots do not show significant motions.

cle acceleration to their static nature, and suggest that they represent shocks in the fluid flow. The radio-bright, static knots at the base of the jet may well be related to a reconfinement shock associated with the transition to trans-sonic flow. However, the fainter knots embedded in the jet flow further out seem more likely to be related to the interaction of the jet with obstacles that are stationary (in practice, $v<0.1 c$ ) in the frame of the host galaxy (cf. [20]). The small size of these obstacles $(\sim 10 \mathrm{pc})$, coupled with the estimated thrust of the jet, gives us only a weak constraint on their mass, which must be greater than a few solar masses. Consequently, there are a number of possible candidates, including WolfRayet stars, but we suggest that the most likely candidates are clumps of cold or warm gas with masses of a few thousand solar masses or more.
There is a large reservoir of cold molecular material in the dust lane of NGC 5128, so this is a realistic scenario. Downstream of these stationary knots, we see regions of bright, relatively diffuse radio emission with little associated $\mathrm{X}$-ray; it is possible that these represent the compression and turbulent particle acceleration that we would expect downstream of an obstacle.

This leaves unanswered the question of what produces the diffuse emission throughout the jet. There is evidence on larger scales (Fig. 3) that the diffuse emission is edge-brightened in places, which, as the edges of the jets are regions of strong shear [1] might mean that it is associated with genuinely diffuse acceleration processes such as second-order Fermi acceleration. Although the jet will naturally contain a large number of normal stars (a few $\times 10^{6}$ in the inner kpc), each of 


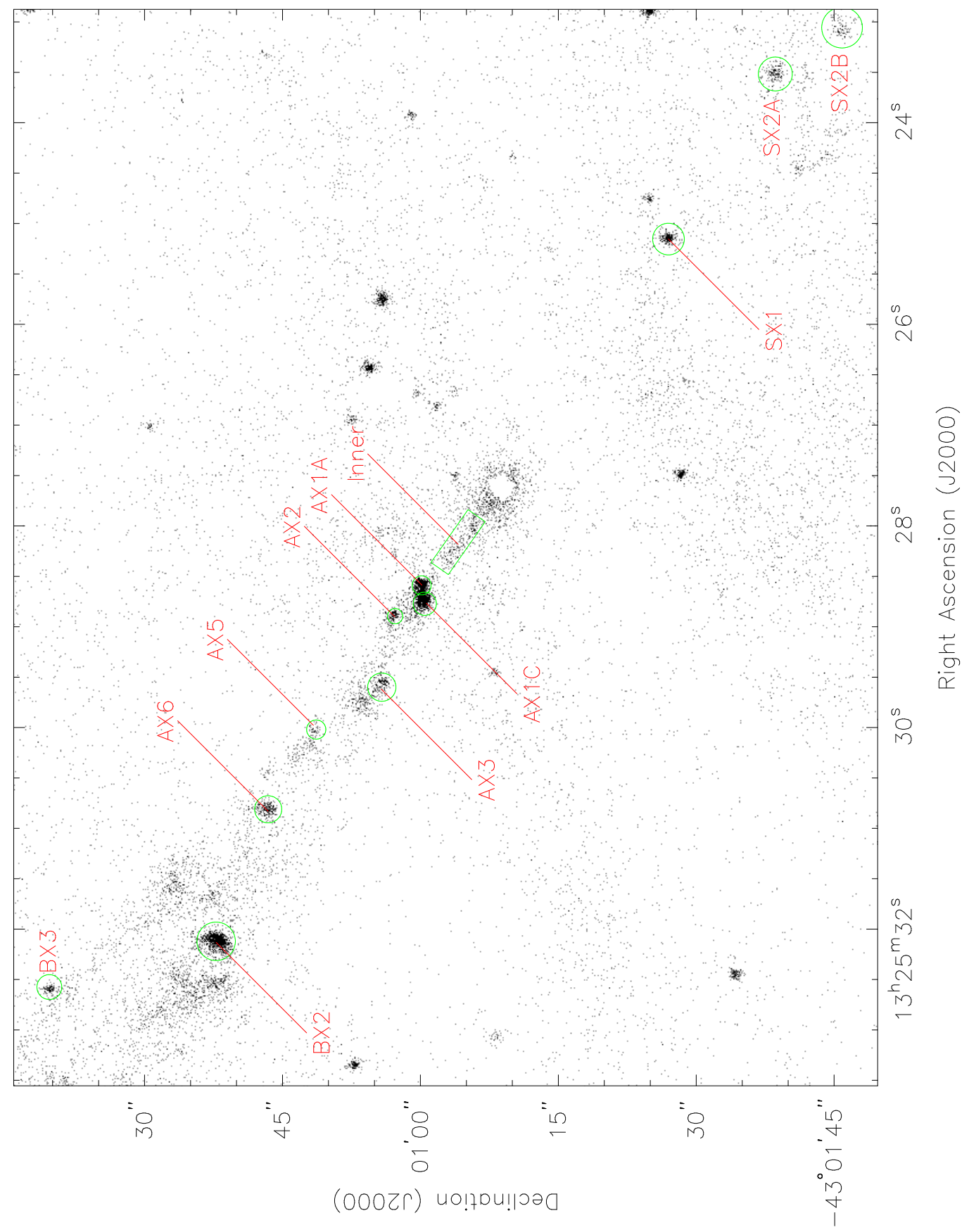

Figure 2. X-ray knots in the jet of Centaurus A. The greyscale shows the raw counts in the energy band $0.4-2.5 \mathrm{keV}$, with 0.123 -arcsec pixels. The active nucleus is almost invisible here because of strong pileup and a high absorption column. 


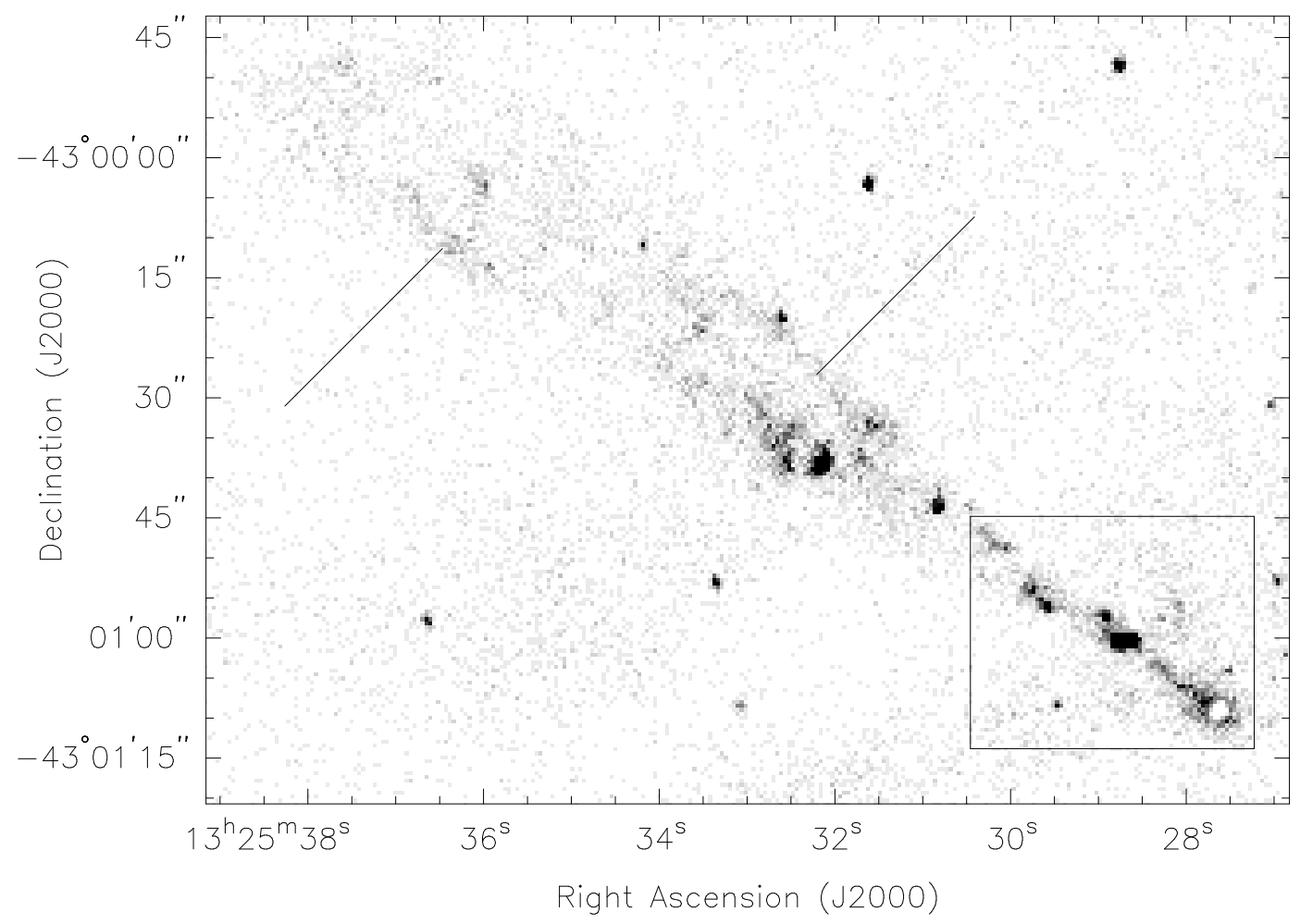

Figure 3. The X-ray structure of the large-scale Cen A jet. The greyscale shows the raw counts in the $0.4-2.5 \mathrm{keV}$ band; the pixels are 0.492 arcsec on a side and black is 12 counts per pixel. The lines indicate two regions where the X-ray jet appears significantly edge-brightened (see the text). The box, shown for orientation purposes only, shows the region of Fig. 1.

which will act, on a smaller scale, like the obstacles we have discussed above, it is easy to show that the cross-sectional area of the jet that they cover, assuming solar mass loss rates $(\dot{M} \approx 4 \times$ $\left.10^{-14} M_{\odot} \mathrm{yr}^{-1}\right)$ and wind speeds $\left(v_{\mathrm{w}} \approx 600 \mathrm{~km}\right.$ $\mathrm{s}^{-1}$ ), is too small to produce the observed bolometric X-ray luminosity in any X-ray jet source, including Cen A, even assuming 100\% energy conversion efficiency. The mean overall stellar mass loss rate is expected to be somewhat higher, but this is dominated by non-main-sequence stars, with lower wind speeds, which do not create a significant obstacle to the jet. If the diffuse emission is a result of interaction with a population of discrete small obstacles, they must (on energetic grounds) block more than about $1 \%$ of the jet's cross-sectional area in the inner $\mathrm{kpc}$, and must also be numerous enough to be unresolved by Chandra; this can be achieved, in the inner kpc of the jet, with a few hundred cold gas clouds with sizes around $1 \mathrm{pc}$.

\section{Variability}

Variability is a key prediction of the synchrotron/particle acceleration models for the Cen A jet, since the synchrotron loss timescales are so short and since the decelerating jet is unlikely to provide a steady flow. There is already some evidence that one of the jet X-ray knots (Fig. 4) 


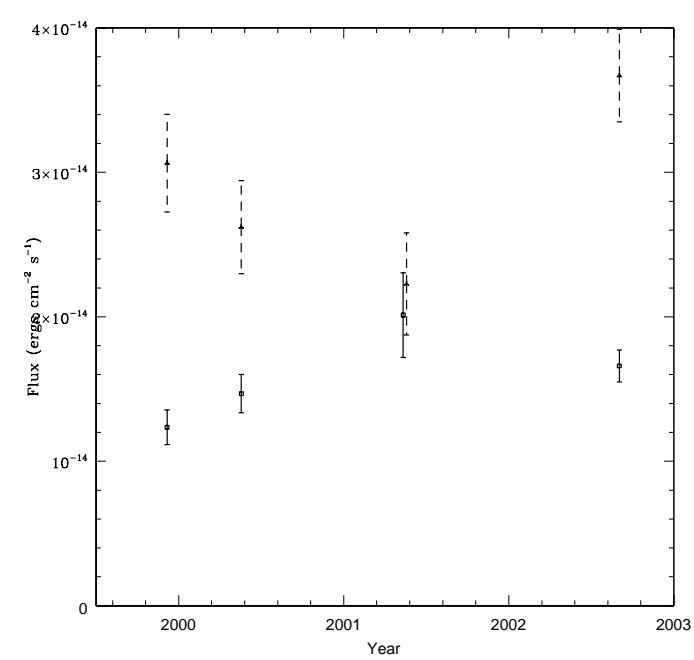

Figure 4. Variability in knot AX6 of the Cen A jet. Solid lines show soft-band flux, dashed lines hard-band.

is variable, and that the hard band is more variable than the soft, as we would expect in a synchrotron model. We will report on the radio and $\mathrm{X}$-ray variability of the jet knots in more detail elsewhere [21].

\section{Summary and outlook}

The evidence available to us is consistent with the idea that a significant fraction of the highenergy particle acceleration in the inner jet of Cen A - and plausibly in other jet sources - is associated with interactions between the jet fluid and stationary or slow-moving obstacles in the flow, although some of the diffuse emission may require a truly non-localized acceleration mechanism. Further monitoring of Cen $\mathrm{A}$ in the radio and X-ray is ongoing; this should allow us to probe the complex relation between particle acceleration and jet dynamics in more detail.

\section{REFERENCES}

1. Laing, R.A., \& Bridle, A.H., 2002a, MNRAS, 336,328

2. Bicknell, G., 1994, ApJ, 422, 542

3. Laing, R.A., \& Bridle, A.H., 2002b, MNRAS, 336,1161

4. Worrall, D.M., Birkinshaw, M., \& Hardcastle, M.J., 2001, MNRAS, 326, L7

5. Hardcastle, M.J., Birkinshaw, M., \& Worrall, D.M., 2001, MNRAS, 326, 1499

6. Schreier, E.J., et al., 1979, ApJ, 234, 39

7. Schreier, E.J., et al., 1982, ApJ, 261, 42

8. Hardcastle, M.J., et al., 2002, MNRAS, 334, 182

9. Harris, D.E., Krawczynski, H., Taylor, G.B., 2002, ApJ, 580, 110

10. Harris, D.E., et al., 2002, ApJ, 580, 110

11. Chiaberge, M., et al. 2003, ApJ, 582, 645

12. Worrall, D.M., Birkinshaw, M., Hardcastle, M.J., 2003, MNRAS, 343, L73

13. Kataoka, J., et al., 2003, A\&A in press (astro$\mathrm{ph} / 0308201$ )

14. Israel, F.P., 1998, ARA\&A, 8, 237

15. Grandi, P., et al., 2003, ApJ, 593, 160

16. Kraft, R.P., et al., 2000, ApJ, 531, L9

17. Kraft, R.P., et al., 2002, ApJ, 569, 54

18. Kraft, R.P., et al., 2003, ApJ, 592, 129

19. Hardcastle, M.J., et al., 2003, ApJ, 593, 169

20. Blandford, R.D., \& Königl, A., 1979, Astrophs. Lett., 20, 15

21. Kraft, R.P., et al., 2004, in preparation. 